\title{
Receiver-Based Flow Control Mechanism with Interlayer Collaboration for Real-Time Communication Quality in W-CDMA Networks ${ }^{\star}$
}

\author{
Hiroyuki Koga ${ }^{1}$, Katsuyoshi Iida ${ }^{1}$, and Yuji Oie ${ }^{2}$ \\ 1 Graduate School of Information Science, \\ Nara Institute of Science and Technology, Japan \\ $\{$ koga, katsu\}@is.naist.jp \\ 2 Department of Computer Science and Electronics, \\ Kyushu Institute of Technology, Japan \\ oie@cse.kyutech.ac.jp
}

\begin{abstract}
Mobile networks are becoming increasingly prevalent, and this has led to an increase in the bandwidth available over wireless links in IMT-2000. Both non-real-time forms of communication, such as email and web browsing, and real-time forms of communication, such as audio and video applications, are well suited to wireless networks. However, wireless networks are subject to relatively long transmission delays because of the need to recover lost packets caused by high bit error rates. This degrades the quality of real-time communications. Therefore, in the present paper, we propose a receiver-based flow control mechanism employing an interlayer collaboration concept to improve the quality of real-time communications without adversely affecting the performance of non-real-time communications on IMT-2000 networks. In addition, simulations are performed in order to evaluate the performance of the proposed mechanism and demonstrate its effectiveness.
\end{abstract}

\section{Introduction}

With the growth of both wireless networks and the Internet, wireless networks are becoming increasingly attractive as an option for data transmission services such as e-mail and web browsing. A next-generation mobile system, International Mobile Telecommunications-2000 (IMT-2000) [1], has been standardized by the International Telecommunication Union (ITU) in order to provide faster data transmission service as well as worldwide roaming capability. WidebandCode Division Multiple Access (W-CDMA) technology, prescribed by the 3rd Generation Partnership Project (3GPP) [2] for wireless networks, is one of the technologies employed by the IMT-2000 system. The current mobile system provides a transmission rate of $9.6 \mathrm{~Kb} / \mathrm{s}$, whereas the $\mathrm{W}$-CDMA system can provide

* This work was supported in part by Grant-in-Aid for the 21st Century COE Program "Ubiquitous Networked Media Computing" and National Institute of Information and Communications Technology. 
a maximum of $384 \mathrm{~Kb} / \mathrm{s}$ outdoors. In addition, the $\mathrm{W}$-CDMA system uses robust error recovery technologies to minimize degradation due to bit errors in wireless channels.

In such W-CDMA networks, Koga et al. investigated how the performance of TCP [3], the transport protocol primarily used for non-real-time communications, is affected by packet losses caused by transmission errors and the ARQ mechanism of Layer 2 and proposed a TCP flow control mechanism to improve TCP performance [4,5]. The proposed mechanism is a receiver-based flow control mechanism that uses information on wireless link conditions provided by an interlayer collaboration concept on a mobile station. This mechanism focuses on TCP performance, in particular, the prevention of throughput degradation due to wireless link conditions, and the stability of the throughput performance.

Furthermore, by increasing the available bandwidth over wireless networks, both real-time communications, such as audio and video applications, and nonreal-time communications become well suited to wireless networks. The coexistence of different types of communication over wireless networks is problematic. In non-real-time communications, importance is attached to efficiency and reliability of data transmission. TCP senders therefore execute flow control so as to use as much of the available bandwidth as possible and to retransmit lost packets [6]. In contrast, real-time communications are more tolerant of packet losses than non-real-time communications but are greatly affected by transmission delays and jitter, i.e., fluctuations in the interarrival times of packets. Therefore, real-time communications generate Constant Bit Rate (CBR) traffic transmitted by UDP datagrams, which do not retransmit lost packets.

Several studies have examined the effect of TCP traffic sharing a link with UDP traffic in wired networks. For example, TCP traffic is known to adversely affect the performance of UDP traffic [7]. Thus, various mechanisms, such as Class Based Queuing (CBQ) 8] and Random Early Drop with In \& Out (RIO) 9], and Resource ReSerVation Protocol (RSVP) [10] have been proposed in order to provide Quality of Service (QoS) assurance for real-time communications. In wireless networks, QoS assurance over wireless networks as well as wired networks must be provided because the available bandwidth of these networks is very different. Although QoS mechanisms in wireless networks have been investigated [11 12/13], very few reports have examined QoS mechanisms for the IMT-2000 system.

In order to provide QoS assurance, particularly in wireless access networks, in which the available bandwidth is generally less stable than in wired networks, the concept of the interlayer collaboration, as discussed in [1415], appears very useful. The interlayer collaboration concept allows an end host, i.e., a mobile station, to control its flow in an intelligent manner without any support by the network. In the present paper, we propose a receiver-based flow control mechanism employing an interlayer collaboration concept to improve the quality of real-time communications without adversely affecting the performance of nonreal-time communications. The basic concept of this mechanism is to limit the bandwidth available for TCP by setting aside adequate bandwidth for UDP 
traffic. The information needed for managing the available bandwidth can be obtained from applications and from the data link layer through an interlayer collaboration concept. Moreover, except for a slight modification to the mobile station, this mechanism does not require any special support at intermediate nodes, including the base station. In addition, modification of the corresponding host is not necessary. Therefore, developing and deploying this mechanism appears very useful.

In the present study, we perform simulations focusing on the characteristics of loss probability and transmission delay time for UDP traffic and the throughput performance for TCP traffic in order to evaluate the effectiveness of the proposed mechanism. We demonstrate that the proposed mechanism achieves performance that is equivalent to the use of priority scheduling at the base station.

\section{Quality of Real-Time Communications in Wireless Networks}

The IMT-2000 system executes retransmissions on Layer 2 in order to minimize degradation due to bit errors. This affects the quality of communications over wireless links. In this section, we describe the W-CDMA system, which is one of the systems employed by the IMT-2000 system, and the major metrics that affect the quality of real-time communications examined herein.

\section{$2.1 \quad$ W-CDMA}

In the W-CDMA environment, the Radio Link Control (RLC) protocol [16] is standardized by $3 \mathrm{GPP}$ as a Layer 2 protocol. The RLC protocol divides a Service Data Unit (SDU), corresponding to an IP datagram, received from the upper layer into several Protocol Data Units (PDUs) of fixed size and transmits them. The RLC protocol employs Selective-Repeat ARQ for recovering lost PDUs. If ARQ is performed repeatedly, the delay becomes infinite. Therefore, SDU discard schemes are defined in order to prevent the delay required for recovering an SDU from reaching infinity. The defined schemes are timer-based discard and number-of-retransmissions-based discard [16], the latter of which is used in the present research [17!18].

In addition, 3GPP specifies a method for preserving the order of packet delivery [19]. We use the scheme whereby some Layer 2 PDUs are kept waiting in Layer 2 of the mobile station until a timeout timer expires. In the present research, an IP datagram is sent to the upper layer if all of the preceding PDUs that were transmitted arrive at the mobile station within the Waiting Timeout $(W T)$ period [5].

\subsection{Metrics Affecting the Quality of Real-Time Communications}

The major metrics that affect the quality of real-time communications, particularly voice communications, are the Signal/Noise $(\mathrm{S} / \mathrm{N})$ ratio, the transmission 
delay time, and packet losses. The $\mathrm{S} / \mathrm{N}$ ratio is determined based on the properties of the codec used for communication, whereas the transmission delay time and packet losses are due primarily to network conditions. Voice communication is interactive and requires a short one-way transmission time between speaker and listener. The Telecommunication Standardization sector of ITU (ITU-T) states in G.114 that a one-way transmission time of less than $150 \mathrm{~ms}$ is acceptable for most user applications and that a one-way transmission time of less than $400 \mathrm{~ms}$ is acceptable for delay-tolerant applications [20]. For Internet communications, a receiver provides a jitter buffer to ensure smooth play. Packets that suffer a transmission delay time that is so large that the buffer cannot remove the effects of jitter will be treated as lost packets. When the loss probability for packets is less than $10 \%$ in networks with short transmission delays, such as wired networks, the quality of communications is good, although it is necessary to lower the loss probability as transmission delay time increases [21.

\section{Proposed Mechanisms}

In wireless networks, retransmission on Layer 2 is performed in order to minimize degradation due to bit errors. This results in a larger transmission delay than that in wired networks, which in turn increases the number of packets treated as lost due to late arrival. Therefore, in order to improve the quality of realtime communications in wireless networks, the transmission delay time must be decreased. In this section, we propose a mechanism by which to improve the quality of real-time communications. In Sect. 3.1, we describe a priority scheduling mechanism, which is commonly used to provide QoS assurance. This mechanism is used in the present study for performance comparison. In Sect. 3.2 we propose a receiver-based flow control mechanism with an interlayer collaboration concept using application layer information and link layer information.

\subsection{Priority Scheduling Mechanism in Layer 2}

One method by which to provide good quality of service is to forward real-time communications packets with higher priority, compared to non-real-time communications packets, at intermediate routers. A static-priority scheduling mechanism [22] has been proposed as a packet scheduling method for such requirements. In the present study, we use the static-priority scheduling mechanism in Layer 2 of the base station, which is used for performance comparison, although such mechanisms are generally used in Layer 3. This is because received SDUs corresponding to the IP datagram are divided into several PDUs of Layer 2 at the base station and retransmissions on Layer 2 are executed in W-CDMA networks, so that even SDUs having priority are not necessarily processed preferentially, even if static-priority scheduling is performed in Layer 3.

This mechanism distinguishes the flow of received packets and gives priority to UDP traffic. When an SDU is divided into several PDUs, the PDUs are stored in a high-priority buffer if the received packet is a UDP packet. TCP packets, 
Table 1. Packet classification for each buffer in the priority scheduling

\begin{tabular}{|c|c|}
\hline Priority & Packet type \\
\hline 1st & Retransmission for UDP \\
2nd & Transmission for UDP \\
3rd & Retransmission for TCP \\
4th & Transmission for TCP \\
\hline
\end{tabular}

however, are stored in a low-priority buffer. Thus, the PDUs for TCP traffic can be transmitted only when the buffer for UDP traffic is empty. The mobile station also has two buffers with different priorities and maintains the sequence integrity of packet delivery for each type of traffic. In addition, each buffer on the base station contains a transmission buffer with a low propriety and a retransmission buffer with a high priority. The former stores newly received PDUs, and the latter stores the PDUs that should be retransmitted due to transmission errors. That is, the base station has four buffers and the mobile station has two buffers. Table 1 summarizes the priority for each type of traffic.

\subsection{Receiver-Based Flow Control Mechanism Employing an Interlayer Collaboration Concept on the Mobile Station}

In this section, we describe the receiver-based flow control mechanism employing an interlayer collaboration concept on the mobile station. In this mechanism, the TCP receiver on the mobile station informs the sender of the bandwidth that is currently available by sending ACK with the advertised window size (awnd), rather than the usual available capacity of its buffer. Note that the awnd based on buffer capacity is used when it is smaller than the awnd calculated from the available bandwidth. Consequently, the TCP sender can maintain a transmission rate that is the minimum value of the congestion window size (cwnd) and an awnd within the limited available bandwidth. Therefore, this mechanism limits the bandwidth available for TCP in order to leave adequate bandwidth for UDP traffic. This mechanism can reduce loss probability and transmission delay time for UDP packets, even if each type of traffic is transmitted through different paths in the networks.

The awnd value can be calculated based on the capacity of the wireless link and the bandwidth currently used by UDP traffic. The capacity of the wireless link, i.e., the bandwidth-delay product of the wireless link, is obtained from Layer 2 on the mobile station through the interlayer collaboration concept, whereas the bandwidth currently used by UDP traffic is obtained from Layer 7 at the mobile station. On Layer 2, the retransmission of the lost PDUs is executed due to bit errors, so that the delay over the wireless link is equal to the worst time $T$ required to transmit one TCP packet to the mobile station and can be expressed as (1) under the conditions that the transmission delay time of the 


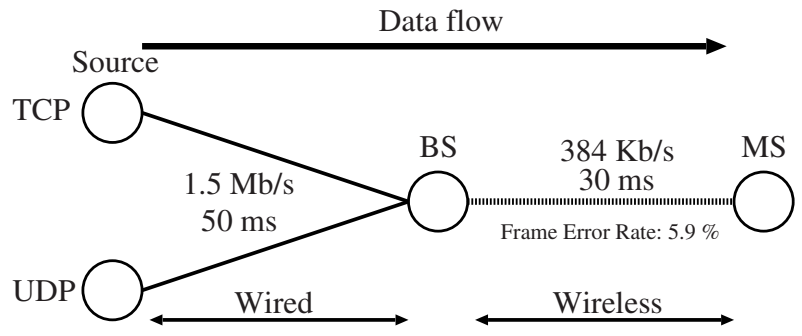

Fig. 1. Simulation model

ACK (NACK) on Layer 2 is ignored.

$$
\begin{aligned}
T= & \left(\frac{\text { TCP packet size }}{\text { available bandwidth }}+2 \times \text { propagation delay }\right) \\
& \times \text { the number of retransmissions. }
\end{aligned}
$$

available bandwidth $=$ link bandwidth obtained from Layer 2

$$
\text { - UDP rate obtained from Layer } 7 .
$$

Using this $T$, the ideal awnd can be obtained by

$$
\text { awnd }=\frac{\text { available bandwidth } \times T}{\text { TCP packet size }} .
$$

The priority scheduling mechanism mentioned in the previous subsection must be implemented on both the base station and the mobile station, which increases processing costs as the number of flows processed at the base station increases. In addition, the type of flow, i.e., TCP or UDP, in Layer 2 must be distinguished. On the other hand, except for a slight modification to the mobile station, the receiver-based flow control employing an interlayer collaboration concept proposed herein does not require any special support at the intermediate nodes, including the base station, and acts to increase the number of flows. In addition, the proposed mechanism does not require any modification to the corresponding host. Therefore, the proposed mechanism is more effective with respect to development and deployment.

\section{Simulation Model}

For the simulation of the present study, we consider a network model in which both real-time communications and non-real-time communications with different transmitting paths on wired links share an IMT-2000 link and focus our examination on the performance of QoS mechanisms. In this section, we describe the simulation model used in the present study. We used the VINT Network Simulator NS Version 2 [23] to which we added modules for Layer 2 ARQ schemes over wireless links. 
In our simulation, real-time communications generating CBR traffic that is transmitted by UDP, e.g., audio and video streaming, share a wireless link with non-real-time communications generating TCP traffic, e.g., file transfer, as shown in Fig. 1 In the W-CDMA system, a dedicated channel is usually used for data transmission. Therefore, we use a simple model that focuses on one Mobile Station (MS) as a receiver. Each source transmits packets from wired links to wireless links. Both wired links have a bandwidth of $1.5 \mathrm{Mb} / \mathrm{s}$ and a propagation delay of $50 \mathrm{~ms}$. The wireless link has a constant bandwidth of $384 \mathrm{~Kb} / \mathrm{s}$ without interference and a link delay of $30 \mathrm{~ms}$, including a propagation delay of $5 \mathrm{~ms}$ and processing delays of $25 \mathrm{~ms}$ in the Base Station (BS) and MS, so as to simplify the simulation model. The BS includes a Radio Network Controller (RNC). We assume that the wireless link is suffering from a burst error caused by Rayleigh fading [24. More specifically, users are walking with an MS, which results in a two-path fading channel with a Doppler frequency of $5 \mathrm{~Hz}$. The physical layer encodes Layer 2 PDUs in a Transmission Time Interval (TTI), during which bit errors occur. When the wireless link has the bandwidth of $384 \mathrm{~Kb} / \mathrm{s}$, the TTI is $20 \mathrm{~ms}$ and the transport block size is $7680 \mathrm{bits}$, according to the specification. The average error rate considered here is realistically $5.9 \%$ for the Frame Error Rate (FER) on Layer 2 with FEC, corresponding to a Bit Error Rate (BER) of $10^{-5}$. We also assume that the ACK and Negative ACK (NACK) are errorfree because they are much smaller than the data frame and their error rates approach zero. The BS stores PDUs in the buffer, while being managed on an SDU basis; i.e., an SDU cannot be partly stored in the buffer. In the following, the buffer will be 50 SDUs in length. The MS keeps some PDUs waiting for $W T$ in order to preserve the sequence integrity of packet delivery, and the $W T$ ranges from 0 to $500 \mathrm{~ms}$.

In this simulation, we consider that TCP traffic is used for greedy file transfers, which continuously transmit infinity data from the sender. The TCP variant employed here is NewReno [25, which is used primarily by computers linked by the Internet. The TCP packet size is set to 1500 bytes, including the IP header 5]. The UDP packet size is set to 260 bytes, including the IP header, and its rates are set to 32,64 , and $128 \mathrm{~Kb} / \mathrm{s}$. The Layer 2 PDU size is set to 42 bytes; the header is 2 bytes and the payload is 40 bytes. In Layer 2, Selective-Repeat ARQ is employed, where the maximum number of allowable retransmissions is set to five for TCP traffic in order to achieve a large throughput [5] and ranges from zero to five for UDP traffic.

The simulation experiments are performed for 60 seconds, and the loss probability for UDP traffic and the average throughput performance of TCP traffic are discussed below. Two types of packet losses can occur in UDP traffic: (1) packets can actually be lost, i.e., a buffer overflow occurs in the BS and the Layer 2 ARQ cannot recover the lost PDUs, and (2) packet losses can occur in MS applications, i.e., packet delivery occurs out-of-order and the transmission delay time exceeds 300 ms. These losses are referred to as transport layer loss and application layer loss, respectively. 
Table 2. Loss probability of UDP and average throughput of TCP with optimum parameters for TCP

\begin{tabular}{|c|c|c|c|c|}
\hline \multirow{2}{*}{$\begin{array}{c}\text { UDP rate } \\
{[\mathrm{Kb} / \mathrm{s}]}\end{array}$} & \multicolumn{3}{|c|}{ Loss probability of UDP [\%] } & \multirow{2}{*}{$\begin{array}{c}\text { TCP throughput } \\
{[\mathrm{Kb} / \mathrm{s}]}\end{array}$} \\
\hline & Transport & Application & Total & \\
\hline 32 & 0.35 & \begin{tabular}{|c|}
93.22 \\
\end{tabular} & 93.57 & 293.29 \\
\hline 64 & 0.37 & 91.72 & 92.09 & 258.98 \\
\hline 128 & 1.32 & 87.16 & 88.48 & 194.75 \\
\hline
\end{tabular}

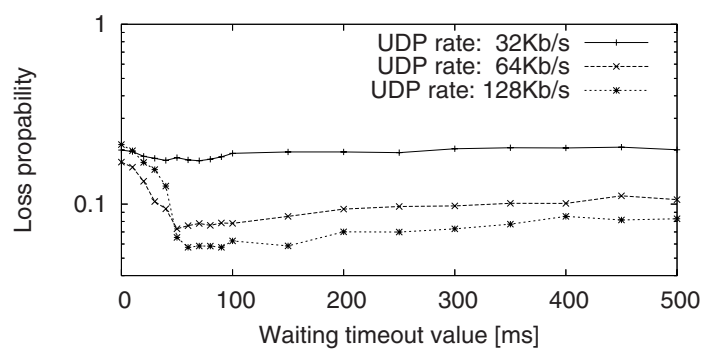

Fig. 2. Effect of $W T$ : no QoS mechanisms

\section{Simulation Results}

In this section, we show the loss probability for UDP packets and the throughput performance of TCP in wireless networks based on our simulation results. We first examine the characteristics of current networks that do not provide QoS assurance for UDP traffic. Then, we show the characteristics when the priority scheduling mechanism and the receiver-based flow control mechanism are employed to improve the loss probability for UDP packets. In addition, we investigate the optimum values of tunable parameters, including the maximum number of allowable retransmissions in Layer 2 ARQ and WT.

\subsection{Case Without QoS Mechanisms}

Before investigating the characteristics for the case in which the QoS mechanisms are employed, we present the results for the case without QoS mechanisms for later comparison. First, we estimate the loss probability for UDP packets when the tunable parameters are set to the optimum values for TCP traffic reported in previous studies 45 . In order to improve TCP throughput performance, the maximum number of allowable retransmissions in Layer 2 ARQ should be set to high values and the WT should be high enough to preserve sequence integrity, even when a large number of retransmissions occurs over a wireless link. Table 2 shows the loss probability for UDP packets and the average throughput of TCP when the maximum number of allowable retransmissions in Layer 2 ARQ is set to five and $W T$ is set to $500 \mathrm{~ms}$. The table shows the two types of loss 


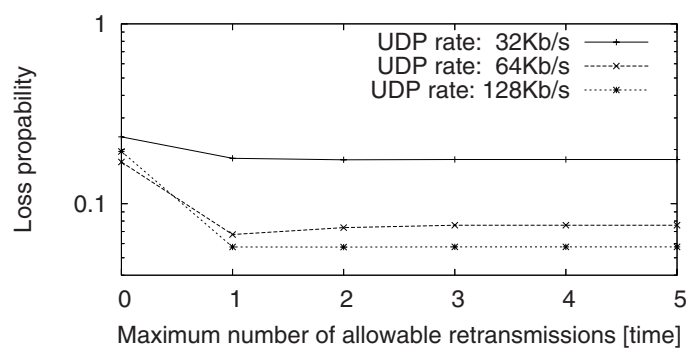

Fig. 3. Effect of maximum number of allowable retransmissions: no QoS mechanisms

probabilities for UDP packets, transport layer loss and application layer loss. All packets of each type of traffic are stored in a single buffer. As Table 2 shows, the loss probability for UDP packets is very high, particularly for application layer loss. This is due to the fact that the transmission delay time very often exceeds $300 \mathrm{~ms}$. Therefore, the transmission delay time should be reduced in order to improve the quality of real-time communications.

Next, we investigate the optimum parameters for decreasing the loss probability for UDP packets. The loss probabilities for UDP packets for the range of $W T$ from 0 to $500 \mathrm{~ms}$ are shown in Fig. 2 The buffer size is set at 10 packets that is optimum value in this scenario. As shown in the figure, the loss probability for UDP is minimal when $W T$ is set to approximately $60 \mathrm{~ms}$. A $W T$ of less than $60 \mathrm{~ms}$ is not sufficient for maintaining the sequence integrity of UDP packets, so that out-of-order packet delivery causes a large loss probability. On the other hand, a WT of greater than $60 \mathrm{~ms}$ can increase the transmission delay time, while maintaining the sequence integrity with higher probability, even if out-of-order delivery occurs in Layer 2. Therefore, a WT of approximately 60 ms minimizes the loss probability as a result of a tradeoff relationship between the transmission delay time and the out-of-order packet delivery when the UDP rate is 64 or $128 \mathrm{~Kb} / \mathrm{s}$. In addition, we found that the $W T$ does not affect loss probability when the UDP rate is $32 \mathrm{~Kb} / \mathrm{s}$. The reason for this is discussed later in this section.

Finally, Fig. 3 shows the loss probabilities for UDP packets for the maximum number of allowable retransmissions for UDP traffic in Layer 2 ARQ ranging from zero to five. The maximum number of allowable retransmissions for TCP traffic is five and $W T$ is set to $60 \mathrm{~ms}$. As shown in Fig. 3 Layer 2 ARQ can improve the loss probability for UDP packets.

The above results indicate that the optimum parameters for UDP are a maximum number of allowable retransmissions of one and a $W T$ of $60 \mathrm{~ms}$. Table 3 summarizes the loss probability of UDP packets and the average throughput of TCP when the parameters are set to these optimum values. Comparison of Tables 2 and 3 reveals that setting parameters to optimum values for UDP traffic greatly reduces application layer losses. However, high-quality real-time commu- 
Table 3. Loss probability of UDP and average throughput of TCP with optimum parameters for UDP

\begin{tabular}{|c|c|c|c|c|}
\hline \multirow{2}{*}{$\begin{array}{l}\text { UDP rate } \\
{[\mathrm{Kb} / \mathrm{s}]}\end{array}$} & \multicolumn{3}{|c|}{ Loss probability of UDP [\%] } & \multirow{2}{*}{$\begin{array}{c}\text { TCP throughput } \\
{[\mathrm{Kb} / \mathrm{s}]}\end{array}$} \\
\hline & Transport & Application & Total & \\
\hline 32 & 3.14 & 14.72 & 17.86 & 277.53 \\
\hline 64 & 3.49 & 3.24 & 6.73 & 241.89 \\
\hline 128 & 5.38 & 0.36 & 5.74 & 185.18 \\
\hline
\end{tabular}

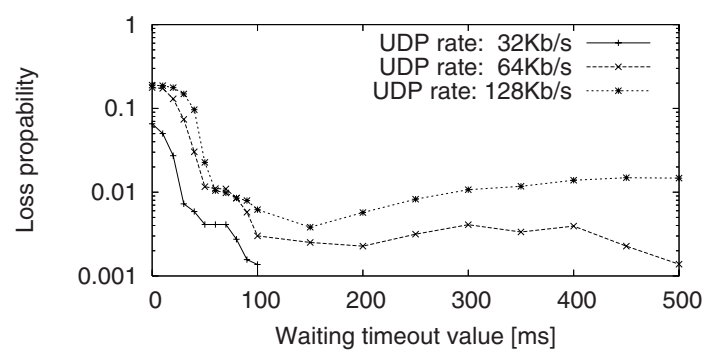

Fig. 4. Effect of $W T$ : priority scheduling mechanism

nications are not yet achieved because of a large loss probability. In addition, TCP throughput performance is degraded and the loss probability for UDP increases as the UDP rate decreases because a lower UDP rate results in an increase in the number of TCP packets occupying the BS buffer. Consequently, the queuing delay for UDP packets increases, with the result that the transmission delay time exceeds $300 \mathrm{~ms}$ and application layer losses occur, particularly when the UDP rate is $32 \mathrm{~Kb} / \mathrm{s}$.

\subsection{Case Employing a Priority Scheduling Mechanism in Layer 2}

In this section, we employ a priority mechanism in Layer 2 in order to decrease the transmission delay time of UDP traffic. As described in Sect. 3.1, the BS has four buffers, and the MS has two buffers with different priorities and maintains the sequence integrity of packet delivery for each type of traffic in Layer 2. The sizes of the Layer 2 buffer and the $W T$ for each type of traffic are assumed to be the same.

Figure 4 shows the loss probabilities for UDP packets for $W T$ ranging from 0 to $500 \mathrm{~ms}$. The maximum number of allowable retransmissions in Layer 2 ARQ is set to five. This is the optimum value for TCP traffic. As the figure shows, the loss probability is greatly reduced when the WT is $150 \mathrm{~ms}$, which is a result of a tradeoff relationship between the transmission delay time and the out-of-order packet delivery, as mentioned in the previous subsection. In this case, WT is larger than that for a case without QoS mechanisms because the transmission delay time of high-priority UDP traffic when using a priority 


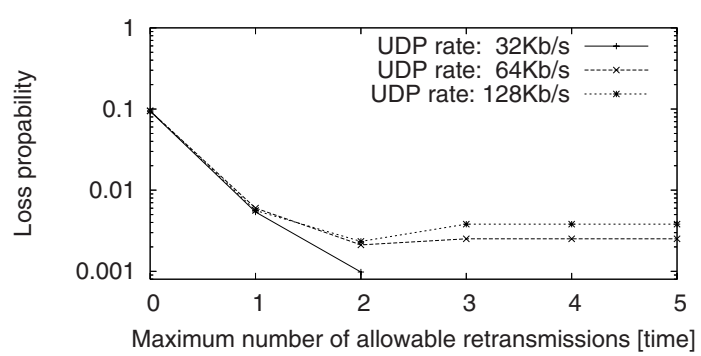

Fig. 5. Effect of maximum number of allowable retransmissions: priority scheduling mechanism

Table 4. Loss probability of UDP and average throughput of TCP with priority scheduling mechanism

\begin{tabular}{|c||c|c|c||c|}
\hline \multirow{2}{*}{$\begin{array}{c}\text { UDP rate } \\
{[\mathrm{Kb} / \mathrm{s}]}\end{array}$} & Loss probability of UDP [\%] & TCP throughput \\
\cline { 2 - 4 } & Transport & Application & Total & $\begin{array}{c}\text { Thb/s }] \\
{[32}\end{array}$ \\
\hline 64 & 0.10 & 0.00 & 0.10 & 294.13 \\
\hline 128 & 0.07 & 0.14 & 0.21 & 261.86 \\
\hline
\end{tabular}

scheduling mechanism becomes smaller, and this allows the $W T$ to be larger in order to maintain the sequence integrity of packet delivery. Next, Fig. 5 shows the loss probability for UDP packets for a $W T$ of $150 \mathrm{~ms}$ and the maximum number of allowable retransmissions in Layer 2 ARQ ranging from zero to five. The figure shows that setting the maximum number of allowable retransmissions to two minimizes the loss probability.

The above results suggest that the optimum parameters for the UDP are a maximum of two allowable retransmissions and a $W T$ of $150 \mathrm{~ms}$. Table 4 summarizes the loss probabilities for UDP packets and the average TCP throughput for the optimum parameters. Using this priority scheduling mechanism in Layer 2 drastically improves the loss probability for UDP packets, providing QoS assurance for real-time communications. Furthermore, TCP throughput is satisfactory.

\subsection{Case Employing a Receiver-Based Flow Control Mechanism}

The priority scheduling mechanism must be implemented in both the BS and the MS, as mentioned above. Since this is highly complex, we employ a receiverbased flow control mechanism using an interlayer collaboration concept, which does not require any special support in the intermediate nodes including the $\mathrm{BS}$, except for a slight modification to the MS, as an alternative mechanism in this section. In this mechanism, instead of the usual available capacity of its buffer, the TCP receiver at the MS informs the sender of the currently available 


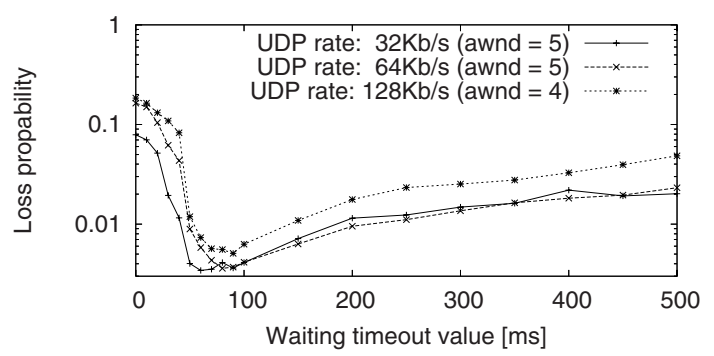

Fig. 6. Effect of $W T$ : receiver-based flow control mechanism

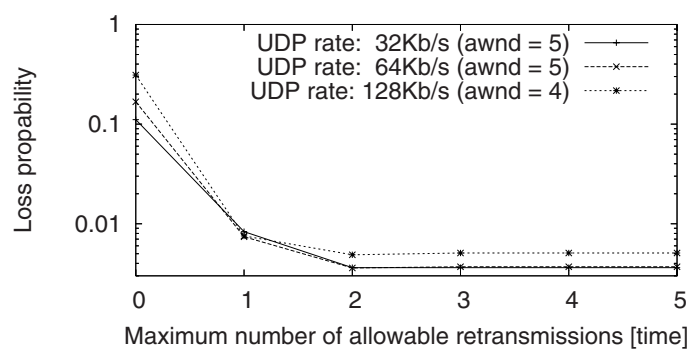

Fig. 7. Effect of maximum number of allowable retransmissions: receiver-based flow control mechanism

bandwidth by sending ACK with an awnd value, in order to limit the bandwidth available for TCP traffic so as to leave adequate bandwidth for UDP traffic, as described in Sect. 3.2 The awnd value can be calculated by (3). When UDP rates are 32,64 , and $128 \mathrm{~Kb} / \mathrm{s}$, the available bandwidths are 352,320 , and 256 $\mathrm{Kb} / \mathrm{s}$, respectively, as can be calculated using the information of the UDP rate obtained from Layer 7 and the bandwidth of the wireless link obtained from Layer 2. In addition, two retransmissions are actually executed in most of the simulation cases because of the assumption in the present study that the FER is $5.9 \%$. Note that the number of retransmissions is obtained from Layer 2 at the MS. Thus, the ideal awnd values become 5.52, 5.20, and 4.56 packets for UDP rates of 32,64 , and $128 \mathrm{~Kb} / \mathrm{s}$, respectively.

Figure [6 shows the loss probability for UDP packets when the awnd is set to five packets for $32 \mathrm{~Kb} / \mathrm{s}$ and $64 \mathrm{~Kb} / \mathrm{s}$ and four packets for $128 \mathrm{~Kb} / \mathrm{s}$, for $W T$ ranging from 0 to $500 \mathrm{~ms}$. The maximum number of retransmissions allowed in Layer $2 \mathrm{ARQ}$ is five. From this figure, we can see that the loss probability is reduced when the $W T$ is approximately $90 \mathrm{~ms}$, which is the tradeoff point mentioned above. Figure 7 shows the loss probability for UDP packets when $W T$ is set to $90 \mathrm{~ms}$ and the maximum number of retransmissions allowed in Layer 2 ranges from zero to five. As this figure shows, the loss probability of UDP traffic reaches a minimum when the maximum number of allowable retransmissions is larger than one. 


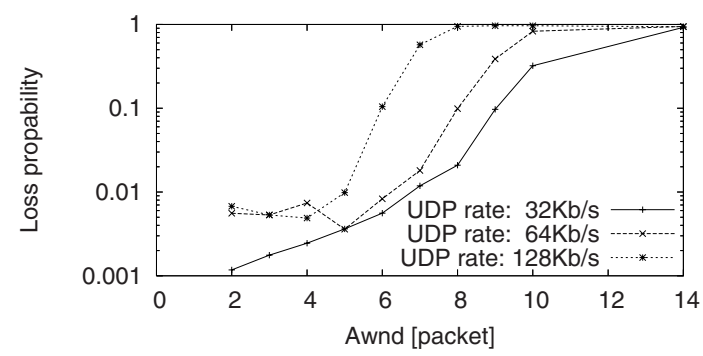

(a) Loss probability of UDP

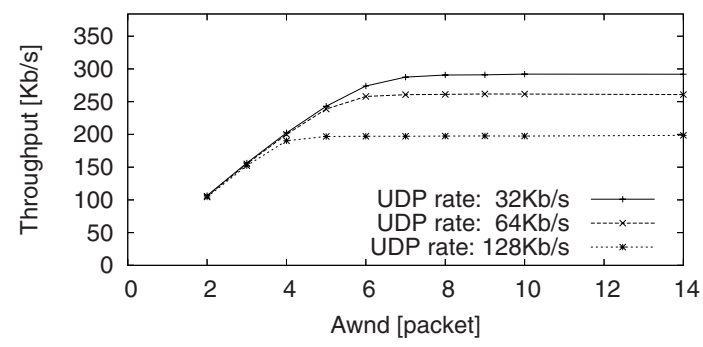

(b) Average throughput of TCP

Fig. 8. Effect of awnd: receiver-based flow control mechanism

Finally, we verify that the calculated awnd value agrees with the measured value. The loss probability for UDP packets and the average throughput of TCP for awnd ranging from 2 to 14 packets are shown in Figs. 8 (a) and (b), respectively. As these figures show, if we consider both loss probability and throughput performance, the optimum awnd values are six packets for $32 \mathrm{~Kb} / \mathrm{s}$, five packets for $64 \mathrm{~Kb} / \mathrm{s}$, and four packets for $128 \mathrm{~Kb} / \mathrm{s}$, which are approximately equal to the calculated values.

The above results indicate that the optimum parameters for UDP are a maximum of two retransmissions allowed, a $W T$ of $90 \mathrm{~ms}$, and awnd values of six, five, and four packets for UDP rates of 32, 64, and $128 \mathrm{~Kb} / \mathrm{s}$, respectively. Table 5 summarizes the loss probability for UDP packets and the average TCP throughput when the parameters are set to these optimum values. These mechanisms reduce the loss probability, providing QoS assurance for real-time communications, and maintaining good TCP throughput performance. 
Table 5. Loss probability of UDP and average throughput of TCP with receiver-based flow control mechanism

\begin{tabular}{|c||c|c|c||c|}
\hline \multirow{2}{*}{$\begin{array}{c}\text { UDP rate } \\
{[\mathrm{Kb} / \mathrm{s}]}\end{array}$} & \multicolumn{2}{|c|}{ Loss probability of UDP [\%] } & TCP throughput \\
\cline { 2 - 4 }$\left[\begin{array}{c}\text { Transport } \\
{[\mathrm{Kb} / \mathrm{s}]}\end{array}\right.$ \\
\hline 64 & 0.02 & 0.54 & 0.56 & 273.98 \\
\hline 128 & 0.07 & 0.29 & 0.36 & 238.74 \\
\hline
\end{tabular}

\section{Conclusions}

In the present study, we have investigated how the quality of real-time communications is affected by non-real-time communications and have determined optimum parameters by which to improve the quality of real-time communications in W-CDMA networks. Through simulations, we found that the loss probability is too large to ensure high-quality real-time communications, even if the parameters associated with W-CDMA systems are set to optimum values for UDP traffic. Therefore, we proposed a receiver-based flow control mechanism employing an interlayer collaboration concept for improving the quality of real-time communications over IMT-2000 wireless networks. In addition, we introduced a priority scheduling mechanism in Layer 2 for comparing the performance of QoS mechanisms. The receiver-based flow control mechanism does not require any special support in the intermediate nodes, including the base station, except for a slight modification to the mobile station. The priority scheduling mechanism, however, must be implemented in both the base station and the mobile station, which increases the processing costs as the number of flows processed at the base station increases. Our simulations indicate that the receiver-based flow control mechanism can achieve comparable improvement in loss probability to that obtained by using a priority scheduling mechanism in Layer 2 of the base station; i.e., the receiver-based flow control mechanism can improve the quality of real-time communications. In addition, the mechanism does not adversely affect TCP throughput performance. Therefore, the receiver-based flow control mechanism employing an interlayer collaboration concept is effective in wireless networks.

\section{References}

1. International Mobile Telecommunications-2000, http://www.imt-2000.org/.

2. 3rd Generation Partnership Project, http://www.3gpp.org/.

3. Stevens, W.R.: TCP/IP Illustrated, Volume 1: The Protocols. Addison-Wesley, Readings, Massachusetts (1994)

4. Koga, H., Kawahara, K., Oie, Y.: TCP flow control using link layer information in mobile networks. In: Proc. SPIE Conference of Internet Performance and Control of Network System III. Volume 4865. (2002) 305-315 
5. Koga, H., Ikenaga, T., Hori, Y., Oie, Y.: Out-of-sequence in packet arrivals due to layer $2 \mathrm{ARQ}$ and its impact on TCP performance in W-CDMA networks. In: Proc. IEEE 2003 Symposium on Applications and the Internet (SAINT 2003). (2003) 398-401

6. Jacobson, V., Karels, M.J.: Congestion avoidance and control. In: Proc. ACM SIGCOMM'88. (1988) 314-329

7. Hori, Y., Sawashima, H., Sunahara, H., Oie, Y.: Performance evaluation of UDP traffic affected by TCP flows. IEICE Transactions on Communications E81-B (1998) 1616-1623

8. Floyd, S., Jacobson, V.: Link-sharing and resource management models for packet networks. IEEE/ACM Transactions on Networking 3 (1995) 365-386

9. Xiao, X., Ni, L.M.: Internet QoS: A big picture. IEEE Network 13 (1999) 8-18

10. Braden, R., Zhang, L., Berson, S., Herzog, S., Jamin, S.: Resource ReSerVation protocol (RSVP). RFC2205 (1997)

11. Vandalore, B., Jain, R., Fahmy, S., Dixit, S.: AQuaFWiN: Adaptive QoS framework for multimedia in wireless networks and its comparison with QoS frameworks. In: Proc. the 24th IEEE Conference on Local Computer Networks. (1999) 88-97

12. Maniatis, S.I., Nikolouzou, E.G., Venieris, I.S.: Convergence of UMTS and Internet services for end-to-end quality of service support. In: Proc. European Wireless 2002. (2002)

13. Marques, V., Aguiar, R.L., Pacyna, P., Fozdecki, J., Beaujean, C., Chaher, N., Gercia, C., Moreno, J.I., Einsiedler, H.: An architecture supporting end-to-end QoS with user mobility for systems beyond 3rd generation. In: Proc. IST Mobile \& Wireless Telecommunications Summit 2002. (2002)

14. Chinta, M., Helal, A., Lee, C.: ILC-TCP: An interlayer collaboration protocol for tcp performance improvement in mobile and wireless environments. In: Proc. the Third IEEE Wireless Communications and Networking Conference (WCNC), New Orleans, Louisiana (2003)

15. Wu, G., Bai, Y., Lai, J., Ogielski, A.: Interactions between TCP and RLP in wireless Internet. In: Proc. IEEE Globecom'99. (1999) 661-666

16. 3GPP TS 25.322 V5.2.0: RLC protocol specification (2002)

17. Tynjala, T., Leppanen, S., Luukkala, V.: Verifying reliable data transmission over UMTS radio interface with high level petri nets. In: Proc. IFIP WG 6.1 International Conference on Formal Techniques for Networked and Distributed Systems (FORTE2002), Lecture Notes in Computer Science. Volume 2529. (2002) 178-193

18. Li, J.: A performance evaluation of the radio link control protocol in 3G UMTS. Diploma thesis, School of Mathematics and Statistics, Carleton University (2003)

19. 3GPP TS 25.301 V4.2.0: Radio interface protocol architecture (2001)

20. ITU-T Rec. G. 114: One-way transmission time (2002)

21. Kostas, T.J., Borella, M.S., Sidhu, I., Schuster, G.M., Grabiec, J., Mahler, J.: Real-time voice over packet-switched networks. IEEE Network 12 (1998) 18-27

22. Siegel, E.D.: Designing Quality of Service. John Wiley \& Sons, Inc. (2000)

23. The Network Simulator ns-2, http://www.isi.edu/nsnam/ns/.

24. Adachi, F., Sawahashi, M., Suda, H.: Wideband DS-CDMS for next generation mobile communications systems. IEEE Communications Magazine 36 (1998) 5669

25. Floyd, S., Henderson, T.: The NewReno modification to TCP's fast recovery algorithm. RFC2582 (1999) 